Pacific

Journal of

Mathematics

\title{
VIRTUAL RATIONAL BETTI NUMBERS OF NILPOTENT-BY-ABELIAN GROUPS
}

BeHRoOZ MirzaiI AND FATEMEH Y. MOKARI 


\title{
VIRTUAL RATIONAL BETTI NUMBERS OF NILPOTENT-BY-ABELIAN GROUPS
}

\author{
BEHROOZ MiRZAII AND FATEMEH Y. MOKARI
}

We study the virtual rational Betti numbers of a nilpotent-by-abelian group $G$, where the abelianization $N / N^{\prime}$ of its nilpotent part $N$ satisfies certain tameness property. More precisely, we prove that if $N / N^{\prime}$ is $2(c(n-1)-1)$ tame as a $G / N$-module, where $c$ is the nilpotency class of $N$, then

$$
\operatorname{vb}_{j}(G):=\sup _{M \in \mathcal{A}_{G}} \operatorname{dim}_{\mathbb{Q}} H_{j}(M, \mathbb{Q})
$$

is finite for all $0 \leq j \leq n$, where $\mathcal{A}_{G}$ is the set of all finite-index subgroups of $G$.

\section{Introduction}

The virtual rational Betti numbers of a finitely generated group studies the growth of the Betti numbers of the group as one follows passage to subgroups of finite index. Following [Bridson and Kochloukova 2015; Kochloukova and Mokari 2015], we define the $n$-th virtual rational Betti number of a finitely generated group $G$ as

$$
\operatorname{vb}_{n}(G):=\sup _{M \in \mathcal{A}_{G}} \operatorname{dim}_{\mathbb{Q}} H_{n}(M, \mathbb{Q}),
$$

where $\mathcal{A}_{G}$ is the set of all subgroups of finite index in $G$.

Bridson and Kochloukova [2015] introduced and studied the first virtual rational Betti number of a finitely generated group $G$ and showed that if $G$ is either a finitely presented nilpotent-by-abelian group or an abelian-by-polycyclic group of type $\mathrm{FP}_{3}$, then $\mathrm{vb}_{1}(G)$ is finite. Moreover, they conjectured that this should be true for all finitely presented soluble groups. As they have shown the finiteness of the first virtual rational Betti numbers of a metabelian group $G$, with normal abelian subgroup $A$ and abelian quotient $Q$ is closely related to the 2-tameness of $A$ as a $Q$-module, an invariant of metabelian groups introduced by Bieri and Strebel [1980].

Mokari is supported by a Capes/CNPq Ph.D. grant.

MSC2010: 20J05, 20J06.

Keywords: virtual Betti numbers, homology of groups, nilpotent-by-abelian groups, nilpotent action. 
Kochloukova and Mokari [2015] extended these results to higher virtual rational Betti numbers of abelian-by-polycyclic groups, by replacing higher tameness with finitely generatedness of high tensor powers of abelian normal subgroups. More precisely, let $A$ be a normal abelian subgroup of $G$ such that the quotient group $Q:=G / A$ is polycyclic. If $Q$ is not abelian, we assume that $G$ is of type $\mathrm{FP}_{3}$. Then it is shown in [Kochloukova and Mokari 2015, Theorem $\mathrm{A}]$ that if $\bigotimes_{\mathbb{Q}}^{2 n}\left(A \otimes_{\mathbb{Z}} \mathbb{Q}\right)$ is finitely generated as a $\mathbb{Q} Q$-module via the diagonal action, then $\operatorname{vb}_{j}(G)$ is finite for $0 \leq j \leq n$. Note that if $G$ is metabelian, then finitely generatedness of $\bigotimes_{\mathbb{Q}}^{2 n}\left(A \otimes_{\mathbb{Z}} \mathbb{Q}\right)$ is equivalent to $2 n$-tameness of $A$ as a $Q$-module (see Theorem 4.1).

Finitely generated soluble groups occurring in applications are often nilpotent -by-abelian-by-finite, that is, any such group $G$ contains subgroups $N \unlhd H \unlhd G$ such that $N$ is nilpotent, $H / N$ abelian and $G / H$ finite. In this paper, we study the virtual rational Betti numbers of nilpotent-by-abelian-by-finite groups. Since $\operatorname{vb}_{n}(G)=\mathrm{vb}_{n}(H)$ (Lemma 5.5), it is sufficient to study virtual rational Betti numbers of nilpotent-by-abelian groups. Here is our main theorem.

Theorem 5.4 (see p. 396). Let $N \longmapsto G \rightarrow Q$ be an exact sequence of groups, where $G$ is finitely generated, $N$ is nilpotent of class $c$ and $Q$ is abelian. If $N / N^{\prime}$ is $2(c(n-1)+1)$-tame, then for any $0 \leq j \leq n, \mathrm{vb}_{j}(G)$ is finite.

As a motivation for the study of virtual rational Betti numbers, one can mention a result of Lück which says that the $L_{2}$-Betti numbers can be computed as a limit involving the ordinary Betti numbers of subgroups of finite index. Here we show that for these groups there is no growth, i.e., the sequences remain bounded. This result therefore confirms Lück's formula by establishing a stronger property for this class of groups [Lück 1994].

To prove our main theorem we needed to study certain aspects of homology of nilpotent groups. Nilpotent groups have a great deal of commutativity built into their structure and they are groups that are "almost abelian". So it is natural to expect that some of the properties of homology of abelian groups, in some way, may be shared by nilpotent groups. In this article, we will study two such properties. For more similarity between homology of abelian and nilpotent groups we refer the interested reader to [Dwyer 1975; Robinson 1976; Hilton et al. 1975].

The $n$-th homology of an abelian group $A$ with rational coefficients is isomorphic to $\bigwedge_{\mathbb{Q}}^{n}\left(A \otimes_{\mathbb{Z}} \mathbb{Q}\right)$. We prove the analogue of this result for nilpotent groups. More precisely, if $N$ is a nilpotent group of class $c$, then we show that there exists a natural filtration of $H_{j}(N, \mathbb{Q})$,

$$
0=E_{0} \subseteq E_{1} \subseteq \cdots \subseteq E_{l-1} \subseteq E_{l}=H_{j}(N, \mathbb{Q}),
$$

such that for any $0 \leq k \leq l, E_{k} / E_{k-1}$ is a natural subquotient of a vector space 
from the set

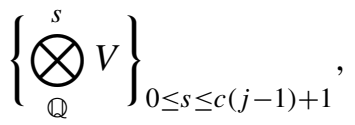

where $V:=\left(N / N^{\prime}\right) \otimes_{\mathbb{Z}} \mathbb{Q}$. When our group is free nilpotent, we show that the above theorem is true even with integral coefficients. Although the existence of the above filtration is not a surprise and can be obtain by easy induction, but the bound $c(j-1)+1$ is new and important for our applications. Furthermore, for groups with small $c$ we show that this bound is sharp. The proofs of these results occupy Sections 1 and 2.

Let $N$ be a nilpotent normal subgroup of a group $G$. If $G$ acts nilpotently on $N / N^{\prime}$, then Theorem 2.1 implies that $G$ acts nilpotently on $H_{k}(N, \mathbb{Q})$. But with a direct method we can prove a more general result. Let $T$ be an $R G$-module, where $R$ is a commutative ring. In Section 3, we will show that if $G$ acts nilpotently on both $N / N^{\prime}$ and $T$, then $G$ acts nilpotently on each $H_{k}(N, T)$ and $H^{k}(N, T)$. As an application, we show that if moreover $G / N$ is finite and $l$-torsion and $1 / l \in R$, then the natural action of $G / N$ on $H_{k}(N, T)$ and $H^{k}(N, T)$ is trivial and therefore the natural maps

$$
\operatorname{corr}_{N}^{G}: H_{k}(N, T) \rightarrow H_{k}(G, T), \quad \operatorname{res}_{N}^{G}: H^{k}(G, T) \rightarrow H^{k}(N, T)
$$

are isomorphisms.

Both of these results about the homology of nilpotent groups are used in the proof of our main theorem (Theorem 5.4).

\section{Differentials of the Lyndon-Hochschild-Serre spectral sequence}

Let $G$ be a group, $A$ an abelian normal subgroup of $G$ and $Q:=G / A$. Let

$$
{ }_{M} \mathcal{E}_{p, q}^{2}=H_{p}\left(Q, H_{q}(A, M)\right) \Rightarrow H_{p+q}(G, M)
$$

be the Lyndon-Hochschild-Serre spectral sequence associated to the exact sequence of groups

$$
A \longmapsto G \rightarrow Q,
$$

where here $M$ is either $\mathbb{Z}$ or $\mathbb{Q}$ with the trivial action of $G$. In this section, we would like to give an explicit formula for the differentials

$$
d_{2, q}^{2}: \mathcal{Q}_{2, q}^{2} \rightarrow{ }_{\mathbb{Q}} \mathcal{E}_{0, q+1}^{2},
$$

for any $q \geq 0$, when $A$ is central, i.e., $A \subseteq Z(G)$.

Let $\phi: A \otimes_{\mathbb{Z}} H_{q}(A, M) \rightarrow H_{q+1}(A, M)$ be the natural product map [Brown 1994, Chapter V, §5], say induced by the shuffle product on the bar resolution, and 
consider the following composition

$$
\begin{gathered}
H^{2}(Q, A) \otimes_{\mathbb{Z}} H_{p}\left(Q, H_{q}(A, M)\right) \stackrel{-\cap-}{\longrightarrow} H_{p-2}\left(Q, A \otimes_{\mathbb{Z}} H_{q}(A, M)\right) \\
\stackrel{-H_{p-2}\left(\mathrm{id}_{Q}, \phi\right)}{\longrightarrow} H_{p-2}\left(Q, H_{q+1}(A, M)\right),
\end{gathered}
$$

where $-\cap-$ is the cap product [Brown 1994, Chapter V, §3].

Let $\rho$ be the element of $H^{2}(Q, A)$ associated to $A \longmapsto G \rightarrow Q$ [Brown 1994, Chapter IV, Theorem 3.12] and set

$$
\Delta(\rho):=H_{p-2}\left(\operatorname{id}_{Q}, \phi\right) \circ(\rho \cap-): H_{p}\left(Q, H_{q}(A, M)\right) \rightarrow H_{p-2}\left(Q, H_{q+1}(A, M)\right) \text {. }
$$

Proposition 1.1 [André 1965, p. 2670]. Let an exact sequence $A \longmapsto G \rightarrow Q$ be given as in above. Then

$$
d_{p, q}^{2}=d_{p, q}^{2}+\Delta(\rho)
$$

where $d_{p, q}^{\prime 2}$ is the differential of the Lyndon-Hochschild-Serre spectral sequence associated to the semidirect product extension $A \longmapsto A \rtimes Q \rightarrow Q$.

Now let $A$ be a central subgroup of $G$. Then the conjugate action of $Q$ on $A$ is trivial and thus $A \rtimes Q=A \times Q$. It is well-known and easy to prove that in this case, for any $p$ and $q, d_{p, q}^{\prime 2}=0$ and therefore

$$
d_{p, q}^{2}=\Delta(\rho) .
$$

Moreover, since $A$ is central, the action of $Q$ on $H_{q}(A, M)$ is trivial. Thus for $M=\mathbb{Q}$, the universal coefficient theorem implies that

$$
\mathbb{Q}_{\mathbb{Q}} \mathcal{E}_{p, q}^{2}=H_{p}(Q, \mathbb{Z}) \otimes_{\mathbb{Z}} H_{q}(A, \mathbb{Q}) \simeq H_{p}(Q, \mathbb{Z}) \otimes_{\mathbb{Z}} \bigwedge_{\mathbb{Q}}^{q}\left(A \otimes_{\mathbb{Z}} \mathbb{Q}\right) .
$$

If $p=2$, then (1-1) finds the following form

$$
H^{2}(Q, A) \otimes_{\mathbb{Z}} H_{2}(Q, \mathbb{Z}) \otimes_{\mathbb{Z}} H_{q}(A, \mathbb{Q}) \stackrel{(-\cap-) \otimes \mathrm{id}}{\longrightarrow} A \otimes_{\mathbb{Z}} H_{q}(A, \mathbb{Q}) \stackrel{\phi}{\rightarrow} H_{q+1}(A, \mathbb{Q}),
$$

where

$$
-\cap-: H^{2}(Q, A) \otimes_{\mathbb{Z}} H_{2}(Q, \mathbb{Z}) \rightarrow A
$$

is the cap product. Therefore from formula (1-2), we obtain the following explicit formula

$$
\begin{aligned}
& d_{2, q}^{2}: \mathcal{Q}_{\mathbb{Q}} \mathcal{E}_{2, q}=H_{2}(Q, \mathbb{Z}) \otimes_{\mathbb{Z}} \bigwedge_{\mathbb{Q}}^{q}\left(A \otimes_{\mathbb{Z}} \mathbb{Q}\right) \rightarrow \mathbb{Q}_{\mathbb{Q}} \mathcal{E}_{0, q+1}^{2}=\bigwedge_{\mathbb{Q}}^{q+1}\left(A \otimes_{\mathbb{Z}} \mathbb{Q}\right), \\
& x \otimes\left(a_{1} \wedge \cdots \wedge a_{q}\right) \mapsto(\rho \cap x) \wedge a_{1} \wedge \cdots \wedge a_{q} .
\end{aligned}
$$

Thus we have proved the following proposition. 
Proposition 1.2. Let $G$ be a group, $A$ a central subgroup of $G$ and $Q:=G / A$. Let

$$
\mathbb{Q}_{\mathcal{Q}, q}^{2}=H_{p}\left(Q, H_{q}(A, \mathbb{Q})\right) \Rightarrow H_{p+q}(G, \mathbb{Q})
$$

be the Lyndon-Hochschild-Serre spectral sequence associated to the extension $A \longmapsto G \rightarrow Q$. Then for any $q \geq 0$, the differential

$$
d_{2, q}^{2}: \mathbb{Q}_{\mathbb{Q}} \mathcal{E}_{2, q}^{2}=H_{2}(Q, \mathbb{Z}) \otimes_{\mathbb{Z}} \bigwedge_{\mathbb{Q}}^{q}\left(A \otimes_{\mathbb{Z}} \mathbb{Q}\right) \rightarrow \mathbb{Q}_{\mathbb{Q}} \mathcal{E}_{0, q+1}^{2}=\bigwedge_{\mathbb{Q}}^{q+1}\left(A \otimes_{\mathbb{Z}} \mathbb{Q}\right),
$$

is given by the formula $x \otimes\left(a_{1} \wedge \cdots \wedge a_{q}\right) \mapsto(\rho \cap x) \wedge a_{1} \wedge \cdots \wedge a_{q}$. Here $\rho$ is the element of $H^{2}(G, A)$ associated to the above extension and the map $-\cap-: H^{2}(Q, A) \otimes_{\mathbb{Z}} H_{2}(Q, \mathbb{Z}) \rightarrow A$ is the cap product. If $A$ is torsion free, then the same result is true for

$$
d_{2, q}^{2}: \mathbb{Z}_{2, q}^{2} \rightarrow \mathbb{Z}_{0, q+1}^{2}
$$

The following corollary will be needed in the next section.

Corollary 1.3. Let $G, A, Q$ and $\mathbb{Q}_{\mathbb{Q}}^{2}$, be as in Proposition 1.2. If $A \subseteq Z(G) \cap G^{\prime}$, then

$$
d_{2, q}^{2}: \mathcal{E}_{2, q}^{2} \rightarrow \mathbb{Q}_{0, q+1}^{2}
$$

is surjective for any $q \geq 0$ and therefore

$$
\mathbb{Q}_{\mathbb{Q}} \mathcal{E}_{0, q}^{\infty}={ }_{\mathbb{Q}} \mathcal{E}_{0, q}^{3}=0 .
$$

Moreover, if A is torsion free, then the same results hold for

$$
d_{2, q}^{2}: \mathbb{Z} \mathcal{E}_{2, q}^{2} \rightarrow \mathbb{Z} \mathcal{E}_{0, q+1}^{2} .
$$

Proof. The spectral sequence ${ }_{M} \mathcal{E}_{p, q}^{2}$, gives us the five term exact sequence

$$
H_{2}(G, M) \rightarrow H_{2}(Q, M) \stackrel{d_{2,0}^{2}}{\longrightarrow} H_{1}(A, M)_{Q} \rightarrow H_{1}(G, M) \rightarrow H_{1}(Q, M) \rightarrow 0,
$$

[Brown 1994, Chapter VII, Corollary 6.4]. Clearly $H_{1}(G, \mathbb{Z}) \simeq H_{1}(Q, \mathbb{Z}) \simeq G / G^{\prime}$. Since the action of $Q$ on $A$ is trivial, we have $H_{1}(A, \mathbb{Z})_{Q} \simeq H_{1}(A, \mathbb{Z})=A$. Thus from the above exact sequence, we obtain the surjective map

$$
d_{2,0}^{2}: H_{2}(Q, \mathbb{Z}) \rightarrow A .
$$

However, from the above, we know that this map is given by the formula $x \mapsto$ $\rho \cap x$. Now by Proposition 1.2, $d_{2, q}^{2}$ is surjective and this immediately implies that $\mathcal{E}_{0, q}^{\infty}=\mathcal{E}_{0, q}^{3}=0$. 


\section{Homology of nilpotent groups}

Let $N$ be a nilpotent group of class $c$ and consider its lower central series,

$$
1=\gamma_{c+1}(N) \subset \gamma_{c}(N) \subset \cdots \subset \gamma_{2}(N) \subset \gamma_{1}(N)=N .
$$

From the exact sequence

$$
\gamma_{c}(N) \longmapsto N \rightarrow N / \gamma_{c}(N),
$$

we obtain the Lyndon-Hochschild-Serre spectral sequence

$$
E_{p, q}^{2}=H_{p}\left(N / \gamma_{c}(N), H_{q}\left(\gamma_{c}(N), T\right)\right) \Rightarrow H_{p+q}(N, T),
$$

where $T$ is an $N$-module.

Since $\gamma_{c+1}(N)=\left[\gamma_{c}(N), N\right]=1$, it follows that $\gamma_{c}(N) \subseteq Z(N)$. So the conjugate action of $N / \gamma_{c}(N)$ on $\gamma_{c}(N)$ is trivial. This also implies that the action of $N / \gamma_{c}(N)$ on $H_{q}\left(\gamma_{c}(N), T\right)$ is trivial, provided that the action of $N$ on $T$ is trivial.

Theorem 2.1. Let $N$ be a nilpotent group of class $c$. Then there exists a natural filtration of $H_{j}(N, \mathbb{Q})$,

$$
0=E_{0} \subseteq E_{1} \subseteq \cdots \subseteq E_{l-1} \subseteq E_{l}=H_{j}(N, \mathbb{Q}),
$$

such that for any $0 \leq k \leq l, E_{k} / E_{k-1}$ is a natural subquotient of a vector space from the set

$$
\left\{\bigotimes_{\mathbb{Q}}^{s} V\right\}_{0 \leq s \leq c(j-1)+1},
$$

where $V:=\left(N / N^{\prime}\right) \otimes_{\mathbb{Z}} \mathbb{Q}$.

Proof. We prove the claim by induction on $c$. All filtrations, homomorphisms and subquotients that will be considered in this proof are natural. If $c=1$, then $N^{\prime}=\gamma_{2}(N)=1$. Thus $N$ is abelian and by [Brown 1994, Theorem 6.4, Chapter V] we have

$$
H_{j}(N, \mathbb{Q}) \simeq\left(\bigwedge_{\mathbb{Z}}^{j} N\right) \otimes_{\mathbb{Z}} \mathbb{Q} \simeq \bigwedge_{\mathbb{Q}}^{j} V .
$$

Clearly $\bigwedge_{\mathbb{Q}}^{j} V$ is of the form $\left(\bigotimes_{\mathbb{Q}}^{j} V\right) / T$, for some subspace $T$ of $\bigotimes_{\mathbb{Q}}^{j} V$. Since $j=1(j-1)+1=c(j-1)+1$, our claim is valid for $c=1$.

Now let $c \geq 2$ and assume that the claim of the theorem is true for all nilpotent groups of class $d, 1 \leq d \leq c-1$. The spectral sequence (2-1) gives us

$$
0=F_{-1} H_{j} \subseteq F_{0} H_{j} \subseteq \cdots \subseteq F_{j-1} H_{j} \subseteq F_{j} H_{j}=H_{j}(N, \mathbb{Q}),
$$

a filtration of $H_{j}(N, \mathbb{Q})$, such that $E_{i, j-i}^{\infty} \simeq F_{i} H_{j} / F_{i-1} H_{j}, \quad 0 \leq i \leq j$. By Corollary $1.3, E_{0, j}^{\infty}=0$, so

$$
F_{0} H_{j}=F_{0} H_{j} / F_{-1} H_{j} \simeq E_{0, j}^{\infty}=0 .
$$


We know that $E_{i, j-i}^{\infty}$ is a subquotient of

$$
E_{i, j-i}^{2} \simeq H_{i}\left(N / \gamma_{c}(N), \mathbb{Q}\right) \otimes_{\mathbb{Q}} H_{j-i}\left(\gamma_{c}(N), \mathbb{Q}\right) .
$$

The group $\gamma_{c}(N)$ is abelian, so

$$
H_{j-i}\left(\gamma_{c}(N), \mathbb{Q}\right) \simeq \bigwedge_{\mathbb{Q}}^{j-i}\left(\gamma_{c}(N) \otimes_{\mathbb{Z}} \mathbb{Q}\right) .
$$

There is a natural surjective map $\bigotimes_{\mathbb{Z}}^{c}\left(N / N^{\prime}\right) \rightarrow \gamma_{c}(N)$, which induces a surjective map

$$
\bigwedge_{\mathbb{Q}}^{j-i}\left(\bigotimes_{\mathbb{Q}}^{c} V\right) \rightarrow \bigwedge_{\mathbb{Q}}^{j-i}\left(\gamma_{c}(N) \otimes_{\mathbb{Z}} \mathbb{Q}\right),
$$

and clearly from this we obtain a surjective map

$$
\bigotimes_{\mathbb{Q}}^{c(j-i)} V \rightarrow H_{j-i}\left(\gamma_{c}(N), \mathbb{Q}\right) .
$$

This implies that $F_{i} H_{j} / F_{i-1} H_{j}$ is a subquotient of

$$
H_{i}\left(N / \gamma_{c}(N), \mathbb{Q}\right) \otimes_{\mathbb{Q}} \bigotimes_{\mathbb{Q}}^{c(j-i)} V .
$$

On the other hand, since $N / \gamma_{c}(N)$ is nilpotent of class $c-1$, by the induction hypothesis, for any $1 \leq i \leq j$, we have a filtration of $H_{i}\left(N / \gamma_{c}(N), \mathbb{Q}\right)$,

$$
0=G_{0, i} \subseteq G_{1, i} \subseteq \cdots \subseteq G_{k_{i}-1, i} \subseteq G_{k_{i}, i}=H_{i}\left(N / \gamma_{c}(N), \mathbb{Q}\right),
$$

such that for any $0 \leq t \leq k_{i}, G_{t, i} / G_{t-1, i}$ is a subquotient of some $\bigotimes_{\overparen{Q}}^{s_{t, i}} V$, where $0 \leq s_{t, i} \leq(c-1)(i-1)+1$. (Note that $\left.\left(N / \gamma_{c}(N)\right) /\left(N / \gamma_{c}(N)\right)^{\prime}=N / N^{\prime}\right)$. This together with (2-3) imply that $F_{i} H_{j} / F_{i-1} H_{j}$ is a subquotient of some $\bigotimes_{\mathbb{Q}}^{s_{i}} V$, where

$$
0 \leq s_{i} \leq(c-1)(i-1)+1+c(j-i)=c(j-1)-i+2 \leq c(j-1)+1 .
$$

This finishes the induction step and so the proof of the theorem.

With some restriction on $N$, one can obtain similar results for integral homology.

Proposition 2.2. Let $N$ be a free nilpotent group of class $c$. Then there exists a natural filtration of $H_{j}(N, \mathbb{Z})$,

$$
0=E_{0} \subseteq E_{1} \subseteq \cdots \subseteq E_{l-1} \subseteq E_{l}=H_{j}(N, \mathbb{Z}),
$$

such that for any $0 \leq k \leq l, E_{k} / E_{k-1}$ is a natural subquotient of a $\mathbb{Z}$-module from the set

$$
\left\{\bigotimes_{\mathbb{Q}}^{s} V\right\}_{0 \leq s \leq c(j-1)+1}, \quad \text { where } V:=N / N^{\prime} \text {. }
$$


Proof. Since $N$ is a free nilpotent group, $\gamma_{c}(N)$ is torsion free. Thus

$$
H_{n}\left(\gamma_{c}(N), \mathbb{Z}\right) \simeq \bigwedge_{\mathbb{Z}}^{n} \gamma_{c}(N)
$$

(see [Brown 1994, Theorem 6.4, Chapter V]) and so it is torsion free. This implies that

$$
E_{i, j-i}^{2} \simeq H_{i}\left(N / \gamma_{c}(N), \mathbb{Z}\right) \otimes_{\mathbb{Z}} H_{j-i}\left(\gamma_{c}(N), \mathbb{Z}\right) .
$$

Now the proof is similar to the proof of Theorem 2.1.

Remark 2.3. We believe that $c(j-1)+1$ is a sharp bound for the existence of a filtration with the above property for $H_{j}(N, \mathbb{Q})$. At least this is true for the extreme cases $c=1$ (abelian $N$ ) or $j=1$ (first homology group case). Also the above proof shows that $E_{1}=F_{1} H_{j}$ is a quotient of

$$
\bigotimes_{\mathbb{Z}}^{c(j-1)+1} V .
$$

This gives an evidence for the fact that the bound $c(j-1)+1$ in Theorem 2.1 is sharp.

Remark 2.4. If $N$ is a nilpotent group of class $c$, then the above theorem also is true for $H_{2}(N, \mathbb{Z})$. By this we mean that there exist a natural filtration of $H_{2}(N, \mathbb{Z})$,

$$
0=E_{0} \subseteq E_{1} \subseteq \cdots \subseteq E_{l-1} \subseteq E_{l}=H_{2}(N, \mathbb{Z}),
$$

such that for any $0 \leq k \leq l, E_{k} / E_{k-1}$ is a natural subquotient of a $\mathbb{Z}$-module from the set

$$
\left\{\bigotimes_{\mathbb{Z}}^{s}\left(N / N^{\prime}\right)\right\}_{0 \leq s \leq c+1} .
$$

This follows from the above proof, using the facts that for an abelian group $A$, $H_{2}(A, \mathbb{Z}) \simeq A \wedge A$ and also for $0 \leq i \leq 2$,

$$
E_{i, 2-i}^{2} \simeq H_{i}\left(N / \gamma_{c}(N), \mathbb{Z}\right) \otimes_{\mathbb{Z}} H_{2-i}\left(\gamma_{c}(N), \mathbb{Z}\right) .
$$

If $c=2$, the complete structure of $H_{2}(N, \mathbb{Z})$ is established in [Kochloukova 1997]. This description is simple if $N$ is torsion-free. In this case $N / \gamma_{2}(N)$ is torsion-free and we obtain a filtration

$$
0 \subseteq F_{1} H_{2} \subseteq F_{2} H_{2}=H_{2}(N, \mathbb{Z})
$$

such that

$$
\begin{aligned}
F_{1} H_{2} & \simeq \frac{\left(N / N^{\prime}\right) \otimes_{\mathbb{Z}} N^{\prime}}{\left\langle x N^{\prime} \otimes[y, z]+y N^{\prime} \otimes[z, x]+z N^{\prime} \otimes[x, y] \mid x, y, z \in N\right\rangle}, \\
F_{2} H_{2} / F_{1} H_{2} & \simeq \operatorname{ker}\left(\left(N / N^{\prime}\right) \wedge\left(N / N^{\prime}\right) \longrightarrow N^{\prime}, x N^{\prime} \wedge y N^{\prime} \mapsto[x, y]\right) .
\end{aligned}
$$


Remark 2.5. Let $N$ be a free nilpotent group of finite rank and of class $c=2$. Then by [Kuz'min and Semenov 1998, p. 532], the differential

$$
d_{p, q}^{2}: E_{p, q}^{2}=\bigwedge_{\mathbb{Z}}^{p}\left(N / N^{\prime}\right) \otimes_{\mathbb{Z}} \bigwedge_{\mathbb{Z}}^{q} N^{\prime} \rightarrow E_{p-2, q+1}^{2}=\bigwedge_{\mathbb{Z}}^{p-2}\left(N / N^{\prime}\right) \otimes_{\mathbb{Z}} \bigwedge_{\mathbb{Z}}^{q+1} N^{\prime}
$$

of the spectral sequence (2-1) is given by the formula

$$
\begin{aligned}
& d_{p, q}^{2}\left(a_{1} N^{\prime} \wedge \cdots \wedge a_{p} N^{\prime} \otimes x_{1} \wedge \cdots \wedge x_{q}\right) \\
& \quad=\sum_{k<l}(-1)^{k+l-1} a_{1} N^{\prime} \wedge \ldots \widehat{a_{k} N^{\prime}} \ldots \widehat{a_{l} N^{\prime}} \cdots \wedge a_{p} N^{\prime} \otimes\left[a_{k}, a_{l}\right] \wedge x_{1} \wedge \cdots \wedge x_{q} .
\end{aligned}
$$

Also in [Kuz'min and Semenov 1998, Theorem 4], it is shown that

$$
H_{j}(N, \mathbb{Z}) \simeq \bigoplus_{i=1}^{j} E_{i, j-i}^{3}
$$

(note that $E_{0, j}^{3}=0$ ). This means that the filtration of $H_{j}(N, \mathbb{Z})$ induced by the spectral sequence,

$$
0=F_{0} H_{j} \subseteq F_{1} H_{j} \subseteq \cdots \subseteq F_{j-1} H_{j} \subseteq F_{j} H_{j}=H_{j}(N, \mathbb{Z}),
$$

has the form

$$
F_{i} H_{j} / F_{i-1} H_{j} \simeq E_{i, j-i}^{3} \subseteq\left(\bigwedge_{\mathbb{Z}}^{i}\left(N / N^{\prime}\right) \otimes_{\mathbb{Z}} \bigwedge_{\mathbb{Z}}^{j-i+1} N^{\prime}\right) / T_{i, j-i},
$$

where $T_{i, j-i}$ is generated by the elements

$$
\sum_{k<l}(-1)^{k+l-1} y_{1} \wedge \cdots \wedge \widehat{y_{k}} \wedge \cdots \wedge \widehat{y_{l}} \wedge \cdots \wedge y_{i+2} \otimes\left[y_{k}, y_{l}\right] \wedge x_{1} \wedge \cdots \wedge x_{j-i-1},
$$

where $y_{h} \in N / N^{\prime}, x_{g} \in N^{\prime}$. This shows that $F_{1} H_{j} \simeq E_{1, j-1}^{3}$ from the filtration is a quotient of $\bigotimes_{\mathbb{Z}}^{2 j-1}\left(N / N^{\prime}\right)$ and is nontrivial. So the bound $2 j-1=c(j-1)+1$ in Theorem 2.1 is sharp.

Corollary 2.6. Let $N \longmapsto G \rightarrow Q$ be an exact sequence of groups, where $N$ is nilpotent of class $c$. Then there exist a natural filtration of $\mathbb{Q} Q$-submodules of $H_{j}(N, \mathbb{Q})$,

$$
0=E_{0} \subseteq E_{1} \subseteq \cdots \subseteq E_{l-1} \subseteq E_{l}=H_{j}(N, \mathbb{Q}),
$$

such that for any $0 \leq k \leq l, E_{k} / E_{k-1}$ is a natural subquotient of a $\mathbb{Q} Q$-module from the set

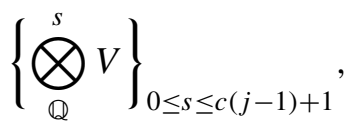

where $V:=\left(N / N^{\prime}\right) \otimes_{\mathbb{Z}} \mathbb{Q}$, and $\bigotimes_{\mathbb{Q}}^{s} V$ is considered as a $\mathbb{Q} Q$-module via the diagonal action of $Q$. 
Proof. We have a natural action of $Q$ on $H_{q}\left(\gamma_{c}(N), \mathbb{Q}\right)$ and $H_{p}\left(N / \gamma_{c}(N), \mathbb{Q}\right)$. From these we obtain a natural action of $Q$ on the Lyndon-Hochschild-Serre spectral sequence

$$
E_{p, q}^{2}=H_{p}\left(N / \gamma_{c}(N), H_{q}\left(\gamma_{c}(N), \mathbb{Q}\right)\right) \Rightarrow H_{p+q}(N, \mathbb{Q}) .
$$

This means that the groups $E_{p, q}^{2}$ are $\mathbb{Q} Q$-modules and the differentials $d_{p, q}^{2}$ are homomorphisms of $\mathbb{Q} Q$-modules. This implies that we have a filtration of $\mathbb{Q} Q$ submodules of $H_{j}(N, \mathbb{Q})$

$$
0=F_{-1} H_{j} \subseteq F_{0} H_{j} \subseteq \cdots \subseteq F_{j-1} H_{j} \subseteq F_{j} H_{j}=H_{j}(N, \mathbb{Q}),
$$

such that each $E_{i, j-i}^{\infty} \simeq F_{i} H_{j} / F_{i-1} H_{j}, 0 \leq i \leq j$, is an isomorphism of $\mathbb{Q} Q$-modules.

It is also easy to see that if $\bigotimes_{\mathbb{Z}}^{c}\left(N / N^{\prime}\right)$ is considered as $\mathbb{Z} Q$-module via the diagonal action of $Q$, then the natural map $\bigotimes_{\mathbb{Z}}^{c}\left(N / N^{\prime}\right) \rightarrow \gamma_{c}(N)$ is a homomorphism of $\mathbb{Z} Q$-modules. Now if we follow the proof of Theorem 2.1, we see that in all steps of the proof the $\mathbb{Q} Q$-structure is preserved. This means that all subquotients considered in the proof of Theorem 2.1 are $\mathbb{Q} Q$-subquotients (i.e., the subquotient structure commutes with the $Q$-action) and the maps are $\mathbb{Q} Q$-homomorphisms, etc. Therefore, as in the proof of Theorem 2.1, we obtain the desired filtration.

\section{Nilpotent action on the homology of nilpotent groups}

We say that a group $G$ acts nilpotently on a $G$-module $T$, if $T$ has a filtration of $G$-submodules

$$
0=T_{0} \subseteq T_{1} \subseteq \cdots \subseteq T_{k-1} \subseteq T_{k}=T,
$$

such that the action of $G$ on each quotient $T_{i} / T_{i-1}$ is trivial.

Corollary 2.6 shows that if $Q=G / N$ acts nilpotently on $N / N^{\prime}$, then it act nilpotently on $H_{j}(N, \mathbb{Q})$ for any $j \geq 0$. This fact can be generalized as follows.

Theorem 3.1. Let $G$ be a group, $N$ a nilpotent normal subgroup of $G$ and let $T$ be a $G$-module. If $G$ acts nilpotently on $N / N^{\prime}$ and $T$, then, for any $k \geq 0, G$ acts nilpotently on $H_{k}(N, T)$ and $H^{k}(N, T)$.

Proof. We prove the claim for the homology functor. The proof for the cohomology functor is similar. The proof is in three steps.

Step 1. $N$ is abelian and $T$ is a trivial $G$-module: Let

$$
0=N_{0} \subseteq N_{1} \subseteq \cdots \subseteq N_{n}=N
$$

be a filtration of $N$ such that $G$ acts trivially on each quotient $N_{i} / N_{i-1}$. We prove this step by induction on the length of the filtration of $N$, i.e., on $n$. If $n=1$, then 
the action of $G$ on $N=N_{1}$ is trivial. So the action of $G$ on $H_{k}(N, T)$ also is trivial. From the exact sequence of groups

$$
N_{1} \longmapsto N \rightarrow N / N_{1},
$$

we obtain the Lyndon-Hochschild-Serre spectral sequence

$$
E_{p, q}^{\prime 2}=H_{p}\left(N / N_{1}, H_{q}\left(N_{1}, T\right)\right) \Rightarrow H_{p+q}(N, T) .
$$

By above, $G$ acts trivially (and so nilpotently) on $H_{q}\left(N_{1}, T\right)$. Since $G / N_{1}$ acts nilpotently on $N / N_{1}$ and $N / N_{1}$ has a filtration of length $n-1$, by induction hypothesis $G / N_{1}$, and so $G$, acts nilpotently on each $E_{p, q}^{\prime 2}$. Since $E_{p, q}^{\prime \infty}$ is a subquotient of $E_{p, q}^{\prime 2}, G$ acts nilpotently on it too. Moreover, $G$ acts naturally on the above spectral sequence which means that each $E_{p, q}^{\prime 2}$ is a $G$-module and the differentials $d_{p, q}^{\prime 2}$ are homomorphisms of $G$-modules. This implies that we have a filtration of $G$-submodules

$$
0=F_{-1} H_{k} \subseteq F_{0} H_{k} \subseteq \cdots \subseteq F_{k-1} H_{k} \subseteq F_{k} H_{k}=H_{k}(N, T),
$$

such that each isomorphism $E_{i, k-i}^{\prime \infty} \simeq F_{i} H_{k} / F_{i-1} H_{k}$ is an isomorphism of $G$ modules. Thus $G$ acts nilpotently on each quotient $F_{i} H_{k} / F_{i-1} H_{k}$. This implies that $G$ acts nilpotently on $H_{k}(N, T)$.

Step 2. $N$ is abelian and $T$ is any $G$-module: Let

$$
0=T_{0} \subseteq T_{1} \subseteq \cdots \subseteq T_{l}=T
$$

be a filtration of $T$, such that $G$ acts trivially on each quotient $T_{i} / T_{i-1}$. In this case we prove the theorem by induction on $l$, the length of the filtration of $T$. If $l=1$, then the action of $G$ on $T=T_{1}$ is trivial, so we arrive at Step 1. From the exact sequence

$$
0 \rightarrow T_{1} \rightarrow T \rightarrow T / T_{1} \rightarrow 0
$$

we obtain the long exact sequence

$$
\cdots \rightarrow H_{k}\left(N, T_{1}\right) \rightarrow H_{k}(N, T) \rightarrow H_{k}\left(N, T / T_{1}\right) \rightarrow \cdots .
$$

We know that $G$ acts nilpotently on $H_{k}\left(N, T_{1}\right)$ and by the induction hypothesis $G$ acts nilpotently on $H_{k}\left(N, T / T_{1}\right)$. Now the above exact sequence implies that $G$ acts nilpotently on $H_{k}(N, T)$.

Step 3. The general case: The proof of this step is by induction on the nilpotent class $c$ of $N$. If $c=1$, then $N$ is abelian and this is done in Step 2. Now assume that the claim is true for all nilpotent groups of class $d, 1 \leq d \leq c-1$. Consider the lower central series of $N$,

$$
1=\gamma_{c+1}(N) \subset \gamma_{c}(N) \subset \cdots \subset \gamma_{2}(N) \subset \gamma_{1}(N)=N .
$$


Note that $\gamma_{c}(N) \subseteq Z(N)$. The exact sequence of groups

$$
\gamma_{c}(N) \longmapsto N \rightarrow N / \gamma_{c}(N),
$$

gives us the Lyndon-Hochschild-Serre spectral sequence

$$
E_{p, q}^{2}=H_{p}\left(N / \gamma_{c}(N), H_{q}\left(\gamma_{c}(N), T\right)\right) \Rightarrow H_{p+q}(N, T) .
$$

We have a natural surjective map

$$
\bigotimes_{\mathbb{Z}}^{c}\left(N / N^{\prime}\right) \rightarrow \gamma_{c}(N),
$$

which is a map of $G$-modules if we consider $\bigotimes_{\mathbb{Z}}^{c}\left(N / N^{\prime}\right)$ as a $G$-module via the diagonal action [Lennox and Robinson 2004, 1.2.11]. Since $G$ acts nilpotently on $N / N^{\prime}$, it also acts nilpotently on $\bigotimes_{\mathbb{Z}}^{c}\left(N / N^{\prime}\right)$. Thus through the above surjective map, $G$ also acts nilpotently on $\gamma_{c}(N)$. By Step 2, $G$ acts nilpotently on $H_{q}\left(\gamma_{c}(N), T\right)$. On the other hand, $N / \gamma_{c}(N)$ is of nilpotent class $c-1$ and $G$ acts nilpotently on $\left(N / \gamma_{c}(N)\right) /\left(N / \gamma_{c}(N)\right)^{\prime} \simeq N / N^{\prime}$. So by the induction hypothesis, $G$ acts nilpotently on each $E_{p, q}^{2}$. Thus $G$ acts nilpotently on each $E_{p, q}^{\infty}$. Finally by the convergence of the spectral sequence, one can show, as in Step 1, that $G$ acts nilpotently on $H_{k}(N, T)$. This completes the proof of the theorem.

If $A$ is an abelian normal subgroup of $G$, then one can show that $G$ is nilpotent if and only if $G / A$ is nilpotent and $G$ acts nilpotently on $A$ [Hilton et al. 1975, Proposition 4.1, Chapter I]. One side of this fact can be generalized as follows.

Corollary 3.2. Let $G$ be a nilpotent group, $N$ a normal subgroup of $G$ and let $T$ be $a G$-module. If $G$ acts nilpotently on $T$, then for any $k \geq 0, G / N$ acts nilpotently on $H_{k}(N, T)$ and $H^{k}(N, T)$.

Proof. Since $G / N^{\prime}$ is nilpotent and $N / N^{\prime}$ is abelian, $G / N^{\prime}$, and so $G$, acts nilpotently on $N / N^{\prime}$. Now the claim follows from Theorem 3.1.

Lemma 3.3. Let $G$ be a finite group, $R$ a commutative ring and $T$ an $R G$-module such that $G$ acts nilpotently.

(i) If $1 /|G| \in R$, then $T$ is a trivial $G$-module.

(ii) If $G$ is nilpotent, $l$-torsion and $1 / l \in R$, then $T$ is a trivial $G$-module.

Proof. (i) We know that the functor $-\otimes_{G} \mathbb{Z}=(-)_{G}$ is right exact. First we show that this is in fact an exact functor if it is considered as a functor from the category of $R G$-modules to the category of $R$-modules. Consider the maps

$$
\begin{aligned}
\alpha_{G}: T^{G} & \rightarrow T_{G}, \quad m \mapsto \bar{m}, \\
\bar{N}: T_{G} & \rightarrow T^{G}, \quad \bar{m} \mapsto N m,
\end{aligned}
$$


where $N:=\sum_{g \in G} g \in R G$. Then clearly $\bar{N} \circ \alpha$ and $\alpha \circ \bar{N}$ coincide with multiplication by $|G|$. Since $1 /|G| \in R, \alpha_{G}$ is an isomorphism. This implies that $(-)_{G}$ is exact, because $(-)^{G}$ is left exact. Next, let

$$
0=T_{0} \subseteq T_{1} \subseteq \cdots \subseteq T_{k}=T
$$

be a filtration of $T$ such that $G$ acts trivially on each $T_{i} / T_{i-1}$. By applying the exact functor $(-)_{G}$ to the exact sequence $0 \rightarrow T_{1} \rightarrow T_{2} \rightarrow T_{2} / T_{1} \rightarrow 0$ and using the fact that $G$ acts trivially on $T_{1}$ and $T_{2} / T_{1}$, we see that

$$
0 \rightarrow T_{1} \rightarrow\left(T_{2}\right)_{G} \rightarrow T_{2} / T_{1} \rightarrow 0
$$

is exact. Therefore $T_{2} \simeq\left(T_{2}\right)_{G}$ and so the action of $G$ on $T_{2}$ is trivial. In a similar way and by induction on $i$, one can show that the action of $G$ on each $T_{i}$ is trivial. Thus the action of $G$ on $T_{k}=T$ is trivial.

(ii) First we prove that $(-)_{G}$ is exact and we do this by induction on the size of $G$. We may assume that $G \neq 1$. Since $G$ is nilpotent, $Z(G) \neq 1$. Let $H$ be a nontrivial cyclic subgroup of $Z(G)$. Then the map $\alpha_{G}$ coincides with the following composition of maps

$$
T^{G} \stackrel{\simeq}{\longrightarrow}\left(T^{H}\right)^{G / H} \stackrel{\alpha_{H}}{\longrightarrow}\left(T_{H}\right)^{G / H} \stackrel{\alpha_{G / H}}{\longrightarrow}\left(T_{H}\right)_{G / H} \stackrel{\simeq}{\longrightarrow} T_{G} .
$$

Now the exactness of the functor $(-)_{G}$ follows from (i) and the induction step. Finally, as in (i) we can prove that $G$ acts trivially on $T$.

Corollary 3.4. Let $G$ be a nilpotent group and $N$ a normal subgroup of $G$ such that $G / N$ is finite and $l$-torsion. Let $R$ be a commutative ring such that $1 / l \in R$ and let $T$ be an $R G$-module. If $G$ acts nilpotently on $T$, then, for any $k \geq 0$, the natural action of $G / N$ on $H_{k}(N, T)$ and $H^{k}(N, T)$ is trivial and therefore the natural maps

$$
\operatorname{corr}_{N}^{G}: H_{k}(N, T) \rightarrow H_{k}(G, T), \quad \operatorname{res}_{N}^{G}: H^{k}(G, T) \rightarrow H^{k}(N, T)
$$

are isomorphisms.

Proof. The claim follows from Corollary 3.2 and Lemma 3.3.

Corollary 3.5. Let $G$ be a nilpotent group and $N$ a subgroup of $G$ such that $G / N$ is finite and $l$-torsion. Let $R$ be a commutative ring such that $1 / l ! \in R$ and let $T$ be an $R G$-module. If $G$ acts nilpotently on $T$, then, for any $k \geq 0$, the natural maps

$$
\operatorname{corr}_{N}^{G}: H_{k}(N, T) \rightarrow H_{k}(G, T), \quad \operatorname{res}_{N}^{G}: H^{k}(G, T) \rightarrow H^{k}(N, T)
$$

are isomorphisms.

Proof. It is well-known that $N$ has a subgroup $L$ such that $L$ is normal in $G$ and $[G: L] \leq[G: N]$ !. Now by Corollary 3.4, the maps

$$
\operatorname{corr}_{L}^{G}: H_{k}(L, T) \rightarrow H_{k}(G, T) \text { and } \operatorname{corr}_{L}^{N}: H_{k}(L, T) \rightarrow H_{k}(N, T)
$$


are isomorphisms. Therefore $\operatorname{corr}_{N}^{G}: H_{k}(N, T) \rightarrow H_{k}(G, T)$ is an isomorphism. The cohomology case can be treated in a similar way.

Example 3.6. In general, in Corollary 3.4 the condition that $[G: N]<\infty$ and $1 / l \in R$ can not be removed. In fact, if $N$ is a noncentral abelian normal subgroup of a nilpotent group $G$, e.g., $G$ a nilpotent group of class $c=3$ and $N=G^{\prime}$, then clearly $G$ does not act trivially on $H_{1}(N, \mathbb{Z})=N$.

\section{Bieri-Strebel invariant}

The main condition of our main Theorem 5.4, proved below, is closely related to an invariant, introduced by Bieri and Strebel [1980], which has played a prominent role in the study of soluble groups which are finitely presented.

Let $Q$ be a multiplicative finitely generated abelian group. A homomorphism of groups

$$
v: Q \rightarrow \mathbb{R}
$$

is called a valuation on $Q$. If $Q$ has rank $n$, then $\operatorname{Hom}_{\mathbb{Z}}(Q, \mathbb{R}) \simeq \mathbb{R}^{n}$, so $\operatorname{Hom}_{\mathbb{Z}}(Q, \mathbb{R})$ can be regarded as a topological vector space. Two valuation $v$ and $v^{\prime}$ on $Q$ are called equivalent if $v^{\prime}=a v$ for some $a \in \mathbb{R}^{>0}$. We denote the equivalence class of $v$ by $[v]$ and the set $S(Q)$ of all equivalence classes of elements of $\operatorname{Hom}_{\mathbb{Z}}(Q, \mathbb{R}) \backslash\{0\}$ is called the valuation sphere, which can be identified with the unit sphere $\mathbb{S}^{n-1} \subset \mathbb{R}^{n}$. Notice that $S(Q)$ is empty precisely when $n=0$, that is, $Q$ is finite. For any valuation $v$ on $Q$ define

$$
Q_{v}:=\{q \in Q \mid v(q) \geq 0\},
$$

which is a submonoid of $Q$.

For a ring $R$, let $R Q_{v}$ be the monoid ring, which clearly is a subring of $R Q$. For a finitely generated $R Q$-module $A$, define

$$
\Sigma_{A}(Q):=\left\{[v] \in S(Q) \mid A \text { is finitely generated over } R Q_{v}\right\} .
$$

A finitely generated $R Q$-module $A$ is called $m$-tame if for any $m$ elements

$$
v_{1}, \ldots, v_{m} \in \operatorname{Hom}_{\mathbb{Z}}(Q, \mathbb{R}) \backslash\{0\}
$$

with $v_{1}+\cdots+v_{m}=0$, there is $1 \leq i \leq m$ such that $\left[v_{i}\right] \in \Sigma_{A}(Q)$.

Theorem 4.1. Let $Q$ be a finitely generated abelian group, $K$ a field, A a finitely generated $K Q$-module and $m \geq 2$ an integer. Then the following statements are equivalent:

(i) A is m-tame as $K Q$-module;

(ii) $\bigotimes_{K}^{m} A$ is finitely generated as $K Q$-module via the diagonal $Q$-action; 
(iii) $\bigotimes_{K}^{i} A$ are finitely generated as $K Q$-modules via the diagonal $Q$-action for $i=2, \ldots, m$;

(iv) $\bigwedge_{K}^{i} A$ are finitely generated as $K Q$-modules via the diagonal $Q$-action for $i=2,3, \ldots, m$;

(v) $\bigwedge_{K}^{m} A$ is finitely generated as $K Q$-module via the diagonal $Q$-action.

Proof. See [Bieri and Groves 1982, Theorem C] and [Kochloukova 1999, Corollary B].

Theorem 4.2. Let $A \longmapsto G \rightarrow Q$ be a short exact sequence of groups with both $A$ and $Q$ abelian and $G$ finitely generated. If $G$ is of type $\mathrm{FP}_{m}$, then $A \otimes_{\mathbb{Z}} K$ is $m$-tame as a $K Q$-module for every field $K$.

Proof. See [Bieri and Groves 1982, Theorem D].

\section{Virtual rational Betti numbers of nilpotent-by-abelian groups}

The following two theorems are taken from [Bridson and Kochloukova 2015] and [Kochloukova and Mokari 2015], respectively, which are very important for the study of virtual rational Betti numbers of abelian-by-polycyclic groups. In this section we will use them for the study of virtual rational Betti numbers of nilpotent-by-abelian groups.

Theorem 5.1 (Bridson-Kochloukova). Let $Q$ be a finitely generated abelian group and $B$ a finitely generated $\mathbb{Q} Q$-module. If $B \otimes_{\mathbb{Q}} B$ is a finitely generated $\mathbb{Q} Q$ module via the diagonal action of $Q$, then

$$
\sup _{M \in \mathcal{A}_{\mathbb{Q}}} \operatorname{dim}_{\mathbb{Q}}\left(B \otimes_{\mathbb{Q} M} \mathbb{Q}\right)<\infty .
$$

Proof. See [Bridson and Kochloukova 2015, Theorem 3.1].

Theorem 5.2 (Kochloukova-Mokari). Let $Q$ be a finitely generated abelian group and $B$ a finitely generated $\mathbb{Q} Q$-module. If $\sup _{m \geq 1} \operatorname{dim}_{\mathbb{Q}}\left(B \otimes_{\mathbb{Q} Q^{m}} \mathbb{Q}\right)<\infty$, then for any $i \geq 0$,

$$
\sup _{m \geq 1} \operatorname{dim}_{\mathbb{Q}} H_{i}\left(Q^{m}, B\right)<\infty .
$$

Proof. See [Kochloukova and Mokari 2015, Theorem 2.4].

Lemma 5.3. Let $Q$ be a finitely generated abelian group. Let $V$ be a $\mathbb{Q} Q$-module such that $\bigotimes_{\mathbb{Q}}^{n} V$ is a finitely generated $\mathbb{Q} Q$-module via the diagonal action of $Q$. If $\sup _{m \geq 1} \operatorname{dim}_{\mathbb{Q}}\left(\left(\bigotimes_{\mathbb{Q}}^{n} V\right) \otimes_{\mathbb{Q} Q^{m}} \mathbb{Q}\right)<\infty$, then for any $\mathbb{Q} Q$-subquotient $U$ of $\bigotimes_{\mathbb{Q}}^{n} V$, we have

$\sup \operatorname{dim}_{\mathbb{Q}}\left(U \otimes_{\mathbb{Q} Q^{m}} \mathbb{Q}\right)<\infty$. 
Proof. First let us assume that $U$ is a quotient of $\bigotimes_{\mathbb{Q}}^{n} V$, i.e., $U=\left(\bigotimes_{\mathbb{Q}}^{n} V\right) / T$, for some $\mathbb{Q} Q$-submodule $T$ of $\bigotimes_{\mathbb{Q}}^{n} V$. Then clearly

$$
\operatorname{dim}_{\mathbb{Q}}\left(U \otimes \mathbb{Q}_{Q^{m}} \mathbb{Q}\right) \leq \operatorname{dim}_{\mathbb{Q}}\left(\left(\bigotimes_{\mathbb{Q}}^{n} V\right) \otimes_{\mathbb{Q} Q^{m}} \mathbb{Q}\right),
$$

and thus

$$
\sup _{m \geq 1} \operatorname{dim}_{\mathbb{Q}}\left(U \otimes_{\mathbb{Q} Q^{m}} \mathbb{Q}\right) \leq \sup _{m \geq 1} \operatorname{dim}_{\mathbb{Q}}\left(\left(\bigotimes_{\mathbb{Q}}^{n} V\right) \otimes_{\mathbb{Q} Q^{m}} \mathbb{Q}\right)<\infty .
$$

Next let $U$ be a $\mathbb{Q} Q$-submodule of some $W:=\left(\otimes_{\mathbb{Q}}^{n} V\right) / T$. Then $W / U$ is of the form $\left(\bigotimes_{\mathbb{Q}}^{n} V\right) / T^{\prime}$ for some $\mathbb{Q} Q$-submodule $T^{\prime}$ of $\bigotimes_{\mathbb{Q}}^{n} V$ and so

$$
\sup _{m \geq 1} \operatorname{dim}_{\mathbb{Q}}\left(W \otimes_{\mathbb{Q} Q^{m}} \mathbb{Q}\right)<\infty, \quad \sup _{m \geq 1} \operatorname{dim}_{\mathbb{Q}}\left((W / U) \otimes_{\mathbb{Q} Q^{m}} \mathbb{Q}\right)<\infty .
$$

Now from the exact sequence $0 \rightarrow U \rightarrow W \rightarrow W / U \rightarrow 0$, we obtain the long exact sequence

$$
\cdots \rightarrow \operatorname{tor}_{1}^{\mathbb{Q} Q^{m}}(W / U, \mathbb{Q}) \rightarrow U \otimes \mathbb{Q} Q^{m} \mathbb{Q} \rightarrow W \otimes \mathbb{Q} Q^{m} \mathbb{Q} \rightarrow(W / U) \otimes \mathbb{Q} Q^{m} \mathbb{Q} \rightarrow 0,
$$

which implies that

$$
\operatorname{dim}_{\mathbb{Q}}\left(U \otimes \mathbb{Q}_{Q^{m}} \mathbb{Q}\right) \leq \operatorname{dim}_{\mathbb{Q}} \operatorname{tor}_{1}^{\mathbb{Q} Q^{m}}(W / U, \mathbb{Q})+\operatorname{dim}_{\mathbb{Q}}\left(W \otimes \mathbb{Q}_{Q^{m}} \mathbb{Q}\right) .
$$

Since $\sup _{m \geq 1} \operatorname{dim}_{\mathbb{Q}}\left((W / U) \otimes_{\mathbb{Q} Q^{m}} \mathbb{Q}\right)<\infty$, by Theorem 5.2 we obtain

$$
\sup _{m \geq 1} \operatorname{dim}_{\mathbb{Q}} H_{i}\left(Q^{m}, W / U\right)<\infty .
$$

$\operatorname{But~tor~}_{i}^{\mathbb{Q} Q^{m}}(W / U, \mathbb{Q})=H_{i}\left(Q^{m}, W / U\right)$, thus by (5-1) and (5-2) we have

$$
\sup _{m \geq 1} \operatorname{dim}_{\mathbb{Q}}\left(U \otimes_{\mathbb{Q} Q^{m}} \mathbb{Q}\right)<\infty .
$$

The next theorem is the main result of this paper.

Theorem 5.4. Let $N \longmapsto G \rightarrow Q$ be an exact sequence of groups, where $G$ is finitely generated, $N$ is nilpotent of class $c$ and $Q$ is abelian. If $N / N^{\prime}$ is $2(c(n-1)+1)$ tame, then for any $0 \leq j \leq n, \mathrm{vb}_{j}(G)$ is finite.

Proof. Let $G_{1}$ be a subgroup of finite index in $G$. Let $Q_{1}$ be the image of $G_{1}$ in $Q$ and $N_{1}:=N \cap G_{1}$. Then clearly $\left[Q: Q_{1}\right]<\infty$, and $\left[N: N_{1}\right]<\infty$. From the associated Lyndon-Hochschild-Serre spectral sequence

$$
E_{p, q}^{2}=H_{p}\left(Q_{1}, H_{q}\left(N_{1}, \mathbb{Q}\right)\right) \Rightarrow H_{p+q}\left(G_{1}, \mathbb{Q}\right)
$$


of the extension $N_{1} \longmapsto G_{1} \rightarrow Q_{1}$, we obtain

$$
\operatorname{dim}_{\mathbb{Q}} H_{j}\left(G_{1}, \mathbb{Q}\right)=\sum_{p=0}^{j} \operatorname{dim}_{\mathbb{Q}} E_{p, j-p}^{\infty} \leq \sum_{p=0}^{j} \operatorname{dim}_{\mathbb{Q}} E_{p, j-p}^{2} .
$$

Since $\left[N: N_{1}\right]<\infty$, by Corollary 3.4 , for any $k \geq 0$, we have

$$
H_{k}\left(N_{1}, \mathbb{Q}\right) \simeq H_{k}(N, \mathbb{Q}) .
$$

Thus $E_{p, q}^{2} \simeq H_{p}\left(Q_{1}, H_{q}(N, \mathbb{Q})\right)$. On the other hand, since $\left[Q: Q_{1}\right]<\infty$, there exists $m \in \mathbb{N}$ such that $\left(Q / Q_{1}\right)^{m}=1$. Hence $Q^{m} \subseteq Q_{1}$. Since $Q_{1} / Q^{m}$ is finite, we have

$$
H_{p}\left(Q_{1}, H_{j-p}(N, \mathbb{Q})\right) \simeq H_{p}\left(Q^{m}, H_{j-p}(N, \mathbb{Q})\right)_{Q_{1} / Q^{m}},
$$

and this implies that

$$
\operatorname{dim}_{\mathbb{Q}} H_{p}\left(Q_{1}, H_{j-p}(N, \mathbb{Q})\right) \leq \operatorname{dim}_{\mathbb{Q}} H_{p}\left(Q^{m}, H_{j-p}(N, \mathbb{Q})\right) .
$$

So to prove the theorem it is sufficient to prove that

$$
\sup _{m \geq 1} \operatorname{dim}_{\mathbb{Q}} H_{p}\left(Q^{m}, H_{j-p}(N, \mathbb{Q})\right)<\infty .
$$

By Corollary 2.6, $H_{j-p}(N, \mathbb{Q})$ has a natural filtration of $\mathbb{Q} Q$-submodules

$$
0=E_{0} \subseteq E_{1} \subseteq \cdots \subseteq E_{l-1} \subseteq E_{l}=H_{j-p}(N, \mathbb{Q}),
$$

such that for any $0 \leq k \leq l, E_{k} / E_{k-1}$ is a natural subquotient of a $\mathbb{Q} Q$-module from the set

$$
\left\{\bigotimes_{\mathbb{Q}}^{s} V\right\}_{0 \leq s \leq c(j-p-1)+1}
$$

where $V:=\left(N / N^{\prime}\right) \otimes_{\mathbb{Z}} \mathbb{Q}$ and $\bigotimes_{\mathbb{Q}}^{s} V$ is considered as a $\mathbb{Q} Q$-module via the diagonal action of $Q$. By Theorem 4.1, $\bigotimes_{\mathbb{Q}}^{s} V$ is a finitely generated $\mathbb{Q} Q$-module for $0 \leq s \leq 2 c(j-p-1)+2$. Thus by Theorem 5.1,

$$
\sup _{m \geq 1} \operatorname{dim}_{\mathbb{Q}}\left(\left(\bigotimes_{\mathbb{Q}}^{s} V\right) \otimes_{\mathbb{Q} Q^{m}} \mathbb{Q}\right)<\infty \quad \text { for } 0 \leq s \leq c(j-p-1)+1 \text {. }
$$

Next, Lemma 5.3 implies that

$$
\sup _{m \geq 1} \operatorname{dim}_{\mathbb{Q}}\left(\left(E_{i} / E_{i-1}\right) \otimes_{\mathbb{Q} Q^{m}} \mathbb{Q}\right)<\infty,
$$

and by induction on $i$, one can show that, for any $1 \leq i \leq j-p$

$\sup \operatorname{dim}_{\mathbb{Q}}\left(E_{i} \otimes_{\mathbb{Q} Q^{m}} \mathbb{Q}\right)<\infty$. 
Therefore

Now by Theorem 5.2 , for any $0 \leq p \leq j$,

$$
\sup _{m \geq 1} \operatorname{dim}_{\mathbb{Q}}\left(H_{j-p}(N, \mathbb{Q}) \otimes_{\mathbb{Q} Q^{m}} \mathbb{Q}\right)=\sup _{m \geq 1} \operatorname{dim}_{\mathbb{Q}}\left(E_{l} \otimes_{\mathbb{Q} Q^{m}} \mathbb{Q}\right)<\infty .
$$

$$
\sup _{m \geq 1} \operatorname{dim}_{\mathbb{Q}} H_{p}\left(Q^{m}, H_{j-p}(N, \mathbb{Q})\right)<\infty .
$$

This completes the proof of the theorem.

Lemma 5.5. Let $G$ be a group and $H$ a subgroup of finite index in $G$. Then $\operatorname{vb}_{n}(G)$ is finite if and only if $\mathrm{vb}_{n}(H)$ is finite. In fact, for any $n \geq 0, \mathrm{vb}_{n}(G)=\mathrm{vb}_{n}(H)$.

Proof. If $H_{0}$ is a subgroup of finite index in $H$, then $\left[G: H_{0}\right]=[G: H]\left[H: H_{0}\right]<\infty$. So $\operatorname{dim}_{\mathbb{Q}} H_{n}\left(H_{0}, \mathbb{Q}\right) \leq \mathrm{vb}_{n}(G)$ and hence

$$
\operatorname{vb}_{n}(H) \leq \operatorname{vb}_{n}(G)
$$

If $G_{0}$ is a subgroup of finite index in $G$, then $\left[G_{0}: G_{0} \cap H\right] \leq[G: H]$. So there is a normal subgroup $N$ of $G_{0}$ such that $N \subseteq G_{0} \cap H$ and $\left[G_{0}: N\right]<\infty$. Since $H_{n}\left(G_{0}, \mathbb{Q}\right) \simeq H_{n}(N, \mathbb{Q})_{G_{0} / N}, \operatorname{dim}_{\mathbb{Q}} H_{n}\left(G_{0}, \mathbb{Q}\right) \leq \operatorname{dim}_{\mathbb{Q}} H_{n}(N, \mathbb{Q})$. Now from $[H: N]<\infty$, it follows that $\operatorname{dim}_{\mathbb{Q}} H_{n}\left(G_{0}, \mathbb{Q}\right) \leq \operatorname{dim}_{\mathbb{Q}} H_{n}(N, \mathbb{Q}) \leq \operatorname{vb}_{n}(H)$. Therefore

$$
\operatorname{vb}_{n}(G) \leq \operatorname{vb}_{n}(H)
$$

Corollary 5.6. Let $G$ be a nilpotent-by-abelian-by-finite group, i.e., we have a chain of subgroups $N \unlhd H \unlhd G$, where $N$ is nilpotent, $H / N$ is abelian and $[G: H]<\infty$. If $N$ is of class $c$ and $H / N^{\prime}$ is of type $\mathrm{FP}_{2 c(n-1)+2}$, then $\mathrm{vb}_{j}(G)$ is finite for any $0 \leq j \leq n$.

Proof. Since $H / N^{\prime}$ is metabelian of type $\mathrm{FP}_{2 c(j-p-1)+2}$, by Theorem 4.2 the $Q$ module $\left(N / N^{\prime}\right) \otimes_{\mathbb{Z}} \mathbb{Q}$ is $2(c(j-p-1)+1)$-tame. Now the claim follows from Lemma 5.5 and Theorem 5.4.

Remark 5.7. Theorem 5.4 and Corollary 5.6 generalize [Bridson and Kochloukova 2015, Theorem 5.3 and Corollary 5.4] to higher homology groups.

For the first virtual rational Betti number we can improve the above result a bit.

Proposition 5.8. Let $N \longmapsto G \rightarrow Q$ be an exact sequence of groups, where $N$ is nilpotent and $Q$ is polycyclic. Let $G / N^{\prime}$ be of type $\mathrm{FP}_{3}$ and let $\bigotimes_{\mathbb{Z}}^{2} N / N^{\prime}$ be finitely generated as $\mathbb{Z} Q$-module via the diagonal action. Then $\mathrm{vb}_{1}(G)$ is finite. 
Proof. Let $G_{1}$ be a normal subgroup of finite index in $G$. Let $Q_{1}$ be the image of the $G_{1}$ in $Q$ and $N_{1}=N \cap G_{1}$. The associated Lyndon-Hochschild-Serre spectral sequence of $N_{1} \longmapsto G_{1} \rightarrow Q_{1}$, i.e.,

$$
E_{p, q}^{2}=H_{p}\left(Q_{1}, H_{q}\left(N_{1}, \mathbb{Q}\right)\right) \Rightarrow H_{p+q}\left(G_{1}, \mathbb{Q}\right),
$$

implies that

$$
\begin{aligned}
\operatorname{dim}_{\mathbb{Q}} H_{1}\left(G_{1}, \mathbb{Q}\right) & \leq \operatorname{dim}_{\mathbb{Q}} E_{0,1}^{2}+\operatorname{dim}_{\mathbb{Q}} E_{1,0}^{2} \\
& =\operatorname{dim}_{\mathbb{Q}} H_{0}\left(Q_{1}, H_{1}\left(N_{1}, \mathbb{Q}\right)\right)+\operatorname{dim}_{\mathbb{Q}} H_{1}\left(Q_{1}, \mathbb{Q}\right) .
\end{aligned}
$$

Since any subgroup of a polycyclic group is polycyclic, by [Kochloukova and Mokari 2015, Lemma 3.2] we have $\operatorname{dim}_{\mathbb{Q}} H_{1}\left(Q_{1}, \mathbb{Q}\right) \leq h(Q)$, where $h(Q)$ is the Hirsch length of $Q$. Since $\left[N: N_{1}\right]<\infty$, by Corollary 3.5 we have $H_{1}\left(N_{1}, \mathbb{Q}\right) \simeq H_{1}(N, \mathbb{Q})$. So to prove the claim it is sufficient to prove that

$$
\sup _{\left.Q: Q_{1}\right]<\infty} \operatorname{dim}_{\mathbb{Q}}\left(N / N^{\prime} \otimes_{Q_{1}} \mathbb{Q}\right)<\infty
$$

Let $A=N / N^{\prime}$ and $H=G / N^{\prime}$ and consider the exact sequence $A \longmapsto H \rightarrow Q$. If we put $A_{0}=[A, H]$ and $Q_{0}=H / A_{0}$ and if we follow the proof of Theorem A in [Kochloukova and Mokari 2015], we obtain

$$
\sup _{\left[Q_{0}: Q_{2}\right]<\infty} \operatorname{dim}_{\mathbb{Q}}\left(A_{0} \otimes Q_{2} \mathbb{Q}\right)<\infty .
$$

From the exact sequence $A_{0} \longmapsto A \rightarrow A / A_{0}$, we obtain the exact sequence

$$
A_{0} \otimes_{Q_{2}} \mathbb{Q} \rightarrow A \otimes_{Q_{2}} \mathbb{Q} \rightarrow\left(A / A_{0}\right) \otimes_{Q_{2}} \mathbb{Q} \rightarrow 0,
$$

which implies that

$$
\operatorname{dim}_{\mathbb{Q}}\left(A \otimes Q_{2} \mathbb{Q}\right) \leq \operatorname{dim}_{\mathbb{Q}}\left(A_{0} \otimes_{Q_{2}} \mathbb{Q}\right)+\operatorname{dim}_{\mathbb{Q}}\left(\left(A / A_{0}\right) \otimes_{Q_{2}} \mathbb{Q}\right) .
$$

Now consider the exact sequence $A / A_{0} \longmapsto Q_{0} \stackrel{\beta}{\rightarrow} Q$ and let $Q_{1}=\beta\left(Q_{2}\right)$. Since the action of $A / A_{0}$ over $A$ is trivial, we have $A \otimes_{Q_{1}} \mathbb{Q} \simeq A \otimes_{Q_{2}} \mathbb{Q}$. Since $A / A_{0}$ is a finitely generated abelian group,

$$
\sup _{\left[Q_{0}: Q_{2}\right]<\infty} \operatorname{dim}_{\mathbb{Q}}\left(\left(A / A_{0}\right) \otimes_{Q_{2}} \mathbb{Q}\right)<\infty .
$$

Therefore from the above relations we have

$$
\sup _{\left[Q: Q_{1}\right]<\infty} \operatorname{dim}_{\mathbb{Q}}\left(A \otimes_{Q_{1}} \mathbb{Q}\right)<\infty .
$$

This completes the proof of the theorem. 
Corollary 5.9. Let $N \longmapsto G \rightarrow Q$ be an exact sequence of groups, where $N$ is nilpotent and $Q$ is nilpotent of class $c \leq 2$. If $G / N^{\prime}$ is of type $\mathrm{FP}_{3}$, then $\mathrm{vb}_{1}(G)$ is finite.

Proof. By Lemma 3.5 in the proof of Corollary B in [Kochloukova and Mokari 2015], $\bigotimes_{\mathbb{Q}}^{2}\left(A_{0} \otimes_{\mathbb{Z}} \mathbb{Q}\right)$ is finitely generated as $\mathbb{Q} Q$-module via the diagonal action, where $A_{0}$ is as in the proof of Proposition 5.8. Now we can proceed as in the proof of Proposition 5.8.

\section{Some examples}

6A. S-arithmetic groups. Unfortunately there is no classification of the nilpotentby-abelian groups of type $\mathrm{FP}_{n}$ even in the case of $n=2$, though the metabelian case was solved in [Bieri and Strebel 1980]. In this case type $\mathrm{FP}_{2}$ turns out to be equivalent to finite presentability. Still in the case of soluble $S$-arithmetic groups there is a complete classification of finite presentability [Abels 1987, Theorem 7.5.2, Remark 4, Chapter VII]. They are finitely presented if and only if are of type $\mathrm{FP}_{2}$. Note that soluble $S$-arithmetic groups are nilpotent-by-abelian-by-finite.

By a theorem of Borel-Serre [Abels 1987, Theorem 0.4.4], any $S$-arithmetic subgroup of a reductive group is of type $\mathrm{FP}_{\infty}$ and thus for such soluble subgroups the result of Corollary 5.6 is true for any $j \geq 0$. But such a result can be proved for other type of $S$-arithmetic groups.

The following example was considered in [Abels and Brown 1987]: Let $p$ be a prime and

$$
\Gamma_{n} \leq \mathrm{GL}_{n+1}(\mathbb{Z}[1 / p]),
$$

where $\Gamma_{n}$ is the group of upper triangular matrices $A$ with $A_{1,1}=1=A_{n+1, n+1}$.

Theorem 6.1. The group $\Gamma_{n}$ is of type $\mathrm{FP}_{n-1}$, but not of type $\mathrm{FP}_{n}$.

Proof. See [Abels and Brown 1987, Theorem A].

Let $N_{n}$ be the subgroup of $\Gamma_{n}$ containing all elements of $\Gamma_{n}$, where the main diagonal contains only entries 1 . Then $N_{n}$ is nilpotent and

$$
Q_{n}=\Gamma_{n} / N_{n} \simeq \mathbb{Z}^{n-1} .
$$

In this case the abelianization $V_{n}=N_{n} /\left[N_{n}, N_{n}\right]$ is isomorphic to $\mathbb{Z}[1 / p]^{n}$, so $V_{n} \otimes_{\mathbb{Z}} \mathbb{Q} \simeq \mathbb{Q}^{n}$ is finite dimensional over $\mathbb{Q}$. Hence all tensor and exterior powers of $V_{n}$ are finitely generated over $\mathbb{Q} Q_{n}$. Thus Theorem 4.1 implies that $V_{n} \otimes_{\mathbb{Z}} \mathbb{Q}$ is $m$-tame for any $m \geq 2$. Now by Theorem 5.4 we obtain the following result.

Proposition 6.2. For any $j \geq 0, \mathrm{vb}_{j}\left(\Gamma_{n}\right)$ is finite. 
6B. Groups of finite torsion-free rank. It is a well-known theorem of Mal'cev that polycyclic groups are nilpotent-by-abelian-by-finite [Lennox and Robinson $2004,3.1 .14]$. On the other hand, for a polycyclic group $G$, the group ring $\mathbb{Z} G$ is (right) noetherian [Lennox and Robinson 2004, 4.2.3] and thus $G$ is of type $\mathrm{FP}_{\infty}$. Now by Corollary 5.6, all virtual rational Betti numbers of $G$ are finite. A direct and much easier proof of this fact is given in [Kochloukova and Mokari 2015, Lemma 3.2]

A polycyclic group is a special case of constructible groups. A soluble group is called constructible if and only if it can be built from the trivial group in finitely many steps by taking descending HNN-extensions and finite extensions. It is wellknown that the class of constructible soluble groups is closed with respect to taking homomorphic images and subgroups of finite index [Baumslag and Bieri 1976, Proposition 2, Theorem 4]. Moreover, they have finite Prüfer rank [Baumslag and Bieri 1976, Section 3.3, Remark 2] and thus are nilpotent-by-abelian-by-finite. The last part follows from the proof of [Robinson 1972, Theorem 10.38]. Furthermore, constructible soluble groups are finitely presented and are of type $\mathrm{FP}_{\infty}$ [Baumslag and Bieri 1976, Proposition 1]. Thus by Corollary 5.6 all virtual rational Betti numbers of these groups are finite.

Kochloukova and the second author gave a good bound for virtual rational Betti numbers of a polycyclic group [Kochloukova and Mokari 2015, Lemma 3.2]. Their proof work even for the larger class of groups of finite torsion-free rank. Polycyclic and constructible groups are of finite Prüfer rank and thus they are of finite torsion-free rank.

A group G, not necessarily soluble, is said to be of finite torsion-free rank if it has a series of subgroups

$$
1=G_{0} \triangleleft G_{1} \triangleleft \cdots \triangleleft G_{n}=G,
$$

such that each nontorsion factor $G_{i} / G_{i-1}$ is infinite cyclic. One can show that the number of infinite cyclic factors is independent of the chosen series (see the proof of [Lennox and Robinson 2004, 1.3.3]) which it is called either the torsion-free rank or the Hirsch number of $G$ and we denote it by $h(G)$.

Proposition 6.3. Let $G$ be a group of finite torsion-free rank. Then for any integer $j \geq 0, \operatorname{dim}_{\mathbb{Q}} H_{j}(G, \mathbb{Q}) \leq\left(\begin{array}{c}h(G) \\ j\end{array}\right)$. In particular,

$$
\mathrm{vb}_{j}(G) \leq\left(\begin{array}{c}
h(G) \\
j
\end{array}\right) .
$$

Proof. The proof is similar to that of the case of polycyclic groups given in [Kochloukova and Mokari 2015, Lemma 3.2]. 


\section{Acknowledgments}

We would like to thank Professor D. H. Kochloukova for introducing the problem to us and for her constructive suggestion during the preparation of this paper. Section 6A was suggested by her. Also, J. R. Groves' proof - different from ours - of the special case of Theorem 2.1 for $c=2$ was made available to us by Kochloukova. We would like to thank both for their help and suggestions.

\section{References}

[Abels 1987] H. Abels, Finite presentability of S-arithmetic groups. Compact presentability of solvable groups, Lecture Notes in Mathematics 1261, Springer, Berlin, 1987. MR Zbl

[Abels and Brown 1987] H. Abels and K. S. Brown, "Finiteness properties of solvable $S$-arithmetic groups: an example", J. Pure Appl. Algebra 44:1-3 (1987), 77-83. MR Zbl

[André 1965] M. André, "Le $d_{2}$ de la suite spectrale en cohomologie des groupes", C. R. Acad. Sci. Paris 260 (1965), 2669-2671. MR Zbl

[Baumslag and Bieri 1976] G. Baumslag and R. Bieri, "Constructable solvable groups", Math. Z. 151:3 (1976), 249-257. MR Zbl

[Bieri and Groves 1982] R. Bieri and J. R. J. Groves, "Metabelian groups of type $(\mathrm{FP})_{\infty}$ are virtually of type (FP)", Proc. London Math. Soc. (3) 45:2 (1982), 365-384. MR Zbl

[Bieri and Strebel 1980] R. Bieri and R. Strebel, "Valuations and finitely presented metabelian groups", Proc. London Math. Soc. (3) 41:3 (1980), 439-464. MR Zbl

[Bridson and Kochloukova 2015] M. R. Bridson and D. H. Kochloukova, "The virtual first Betti number of soluble groups", Pacific J. Math. 274:2 (2015), 497-510. MR Zbl

[Brown 1994] K. S. Brown, Cohomology of groups, Graduate Texts in Mathematics 87, Springer, New York, NY, 1994. MR Zbl

[Dwyer 1975] W. G. Dwyer, "Vanishing homology over nilpotent groups", Proc. Amer. Math. Soc. 49 (1975), 8-12. MR Zbl

[Hilton et al. 1975] P. J. Hilton, G. Mislin, and J. Roitberg, Localization of nilpotent groups and spaces, North-Holland Mathematics Studies 15, North-Holland, Amsterdam, 1975. MR Zbl

[Kochloukova 1997] D. H. Kochloukova, The $\mathrm{FP}_{m}$-conjecture for a class of metabelian groups and related topics, Ph.D. thesis, Cambridge University, 1997.

[Kochloukova 1999] D. H. Kochloukova, "Finite generation of exterior and symmetric powers", Math. Proc. Cambridge Philos. Soc. 125:1 (1999), 21-29. MR Zbl

[Kochloukova and Mokari 2015] D. H. Kochloukova and F. Y. Mokari, "Virtual rational Betti numbers of abelian-by-polycyclic groups", J. Algebra 443 (2015), 75-98. MR Zbl

[Kuz'min and Semenov 1998] Y. V. Kuz'min and Y. S. Semenov, “О гомологиях свободной нильпотентной группы класса 2", Mat. Sb. 189:4 (1998), 49-82. Translated as “On the homology of a free nilpotent group of class 2" in Sb. Math. 189:4 (1998), 527-560. MR Zbl

[Lennox and Robinson 2004] J. C. Lennox and D. J. S. Robinson, The theory of infinite soluble groups, Oxford University Press, 2004. MR Zbl

[Lück 1994] W. Lück, "Approximating $L^{2}$-invariants by their finite-dimensional analogues", Geom. Funct. Anal. 4:4 (1994), 455-481. MR Zbl

[Robinson 1972] D. J. S. Robinson, Finiteness conditions and generalized soluble groups, part 2, Ergebnisse der Mathematik und ihrer Grenzgebiete 63, Springer, Berlin, 1972. MR Zbl 
[Robinson 1976] D. J. S. Robinson, "The vanishing of certain homology and cohomology groups", J. Pure Appl. Algebra 7:2 (1976), 145-167. MR Zbl

Received August 30, 2015.

BEHROOZ MIRZAII

Instituto de CiÊNCIAs MATEMÁtiCAS E DE COMPUTAÇÃo (ICMC)

UNIVERSIDADE DE SÃo PAULO

AVENIDA TRABALHADOR SÃO-CARLENSE, 400-CENTRO

13566-590 SÃO CARLOS-SP

BRAZIL

bmirzaii@icmc.usp.br

FATEMEH Y. MOKARI

Instituto de Matemática, EstatísticA E ComputaÇão CientífiCA (IMECC)

UNIVERSIDADE ESTADUAL DE CAMPINAS

RUA SÉRgIO BUARQUE DE HOLANDA, 651

13083-859 CAMPINAS-SP

BRAZIL

f.mokari61@gmail.com 


\title{
PACIFIC JOURNAL OF MATHEMATICS
}

Founded in 1951 by E. F. Beckenbach (1906-1982) and F. Wolf (1904-1989)

$$
\text { msp.org/pjm }
$$

\section{EDITORS}

\author{
Don Blasius (Managing Editor) \\ Department of Mathematics \\ University of California \\ Los Angeles, CA 90095-1555 \\ blasius@math.ucla.edu
}

\author{
Paul Balmer \\ Department of Mathematics \\ University of California \\ Los Angeles, CA 90095-1555 \\ balmer@math.ucla.edu \\ Robert Finn \\ Department of Mathematics \\ Stanford University \\ Stanford, CA 94305-2125 \\ finn@math.stanford.edu \\ Sorin Popa \\ Department of Mathematics \\ University of California \\ Los Angeles, CA 90095-1555 \\ popa@math.ucla.edu
}

\author{
Vyjayanthi Chari \\ Department of Mathematics \\ University of California \\ Riverside, CA 92521-0135 \\ chari@math.ucr.edu \\ Kefeng Liu \\ Department of Mathematics \\ University of California \\ Los Angeles, CA 90095-1555 \\ liu@math.ucla.edu \\ Igor Pak \\ Department of Mathematics \\ University of California \\ Los Angeles, CA 90095-1555 \\ pak.pjm@gmail.com \\ Paul Yang \\ Department of Mathematics \\ Princeton University \\ Princeton NJ 08544-1000 \\ yang@math.princeton.edu
}

\section{PRODUCTION}

Silvio Levy, Scientific Editor, production@msp.org

\section{SUPPORTING INSTITUTIONS}

ACADEMIA SINICA, TAIPEI

CALIFORNIA INST. OF TECHNOLOGY

STANFORD UNIVERSITY

UNIV. OF BRITISH COLUMBIA

UNIV. OF CALIFORNIA, BERKELEY

UNIV. OF CALIFORNIA, DAVIS

UNIV. OF CALIFORNIA, LOS ANGELES

UNIV. OF CALIFORNIA, RIVERSIDE

UNIV. OF CALIFORNIA, SAN DIEGO

UNIV. OF CALIF., SANTA BARBARA
KEIO UNIVERSITY

MATH. SCIENCES RESEARCH INSTITUTE

NEW MEXICO STATE UNIV.

OREGON STATE UNIV.
Daryl Cooper

Department of Mathematics

University of California

Santa Barbara, CA 93106-3080 cooper@math.ucsb.edu

Jiang-Hua Lu

Department of Mathematics

The University of Hong Kong

Pokfulam Rd., Hong Kong

jhlu@maths.hku.hk

$$
\text { Jie Qing }
$$

Department of Mathematics

University of California

Santa Cruz, CA 95064

qing@ cats.ucsc.edu

\author{
UNIV. OF CALIF., SANTA CRUZ \\ UNIV. OF MONTANA \\ UNIV. OF OREGON \\ UNIV. OF SOUTHERN CALIFORNIA \\ UNIV. OF UTAH \\ UNIV. OF WASHINGTON \\ WASHINGTON STATE UNIVERSITY
}

These supporting institutions contribute to the cost of publication of this Journal, but they are not owners or publishers and have no responsibility for its contents or policies.

See inside back cover or msp.org/pjm for submission instructions.

The subscription price for 2016 is US $\$ 440 /$ year for the electronic version, and \$600/year for print and electronic.

Subscriptions, requests for back issues and changes of subscriber address should be sent to Pacific Journal of Mathematics, P.O. Box 4163, Berkeley, CA 94704-0163, U.S.A. The Pacific Journal of Mathematics is indexed by Mathematical Reviews, Zentralblatt MATH, PASCAL CNRS Index, Referativnyi Zhurnal, Current Mathematical Publications and Web of Knowledge (Science Citation Index).

The Pacific Journal of Mathematics (ISSN 0030-8730) at the University of California, c/o Department of Mathematics, 798 Evans Hall \#3840, Berkeley, CA 94720-3840, is published twelve times a year. Periodical rate postage paid at Berkeley, CA 94704, and additional mailing offices. POSTMASTER: send address changes to Pacific Journal of Mathematics, P.O. Box 4163, Berkeley, CA 94704-0163.

PJM peer review and production are managed by EditFLOW ${ }^{\circledR}$ from Mathematical Sciences Publishers.

PUBLISHED BY

\section{I. mathematical sciences publishers}

nonprofit scientific publishing

http://msp.org/

(C) 2016 Mathematical Sciences Publishers 


\section{PACIFIC JOURNAL OF MATHEMATICS}

Volume $283 \quad$ No. $2 \quad$ August 2016

The fundamental theorem of tropical differential algebraic geometry

FUENSANTA AROCA, CRISTHIAN GARAY and ZEINAB TOGHANI

A simple solution to the word problem for virtual braid groups

271

PaOlo Bellingeri, Bruno A. Cisneros de la CruZ and Luis

PARIS

Completely contractive projections on operator algebras

DAVID P. BLECHER and MATTHEW NEAL

Invariants of some compactified Picard modular surfaces and applications

AMIR DŽAMBIĆ

Radial limits of bounded nonparametric prescribed mean curvature surfaces

MOZHGAN (NORA) ENTEKHABI and KIRK E. LANCASTER

A remark on the Noetherian property of power series rings

BYUNG GYUN KANG and PHAN THANH TOAN

Curves with prescribed intersection with boundary divisors in moduli spaces of curves

\section{XIAO-LEI LIU}

Virtual rational Betti numbers of nilpotent-by-abelian groups

BEHROOZ MiRZAII and FATEMEH Y. MOKARI

A planar Sobolev extension theorem for piecewise linear homeomorphisms 405

EMANUELA RADICI

A combinatorial approach to Voiculescu's bi-free partial transforms

PAUL SKOUFRANIS

Vector bundle valued differential forms on $\mathbb{N} Q$-manifolds

LUCA VitAGLiANO

Discriminants and the monoid of quadratic rings 